\title{
Comparison between Natural Drainage Group and Negative Drainage Group after Cervical Laminoplasty: A retrospective STROBE-compliant cohort study
}

\section{Fulin Guan}

The 1st affiliated hospital of Harbin medical university

\section{Hongna Yin}

2nd affiliated hospital of heilongjiang university of chinese medicine

\section{Lin Zhu}

Harbin Medical University

\section{Zhizhuang Zhang}

1 st affiliated hospital of harbin medical university

\section{Qichang Gao}

1st affiliated hospital of harbin medical university

\section{Tuo Shao}

1st affiliated hospital of harbin medical university

\section{Weilong Tang}

1st affiliated hospital of harbin medical university

\section{Guofa Guan}

1st affiliated hospital of harbin medical university

\section{Ming Chen}

1st affiliated hospital of harbin medical university

\section{Zhiyong Chi}

1st affiliated hospital of harbin medical university

Jiaao Gu ( $\square$ jiaaogu@outlook.com )

The 1st affiliated hospital of harbin medical university https://orcid.org/0000-0002-2823-1447

\section{Zhange Yu}

1st affiliated hospital of harbin medical university

\section{Research article}

Keywords: Retract

Posted Date: December 4th, 2019 
DOI: https://doi.org/10.21203/rs.2.15671/v2

License: (c) (1) This work is licensed under a Creative Commons Attribution 4.0 International License. Read Full License 
The authors have withdrawn this preprint from Research Square 